\title{
Las relaciones universidad-comunidad: un espacio de reconfiguración de lo público ${ }^{1}$
}

\author{
Graciela Tonon \\ Universidad Nacional de Lomas de Zamora, Buenos Aires, Argentina. \\ Email: gracielatonon@hotmail.com
}

\begin{abstract}
Resumen: El artículo desarrolla una primera propuesta para abordar el tema de la relación universidad-comunidad como un espacio de reconfiguración de lo público, explicitando en primer lugar una posición teórica acerca de los conceptos espacio público, comunidad y universidad, comunidad, para poder avanzar luego en las posibles relaciones entre los mismos. Señala los cambios acontecidos en el siglo XXI en relación a la reconfiguración del espacio público ya no exclusivamente ligado al Estado sino que como espacio de participación ciudadana y comunitaria; proponiendo una modalidad de trabajo de la universidad en sus relaciones con la comunidad, que coloca a los ciudadanos como protagonistas desde la idea de democratización del saber.
\end{abstract}

Palabras clave: universidad, comunidad, relaciones, espacio público

\section{University-community relations: a zone of reconfiguration of public space}

\begin{abstract}
This paper develops a first proposal to address the issue of university-community as an area of reconfiguration of the public, explaining first a theoretical position about the concepts: public space, community and university community, to advance then to the possible relationships between them. It points out the changes of the XXI century in relation to the reconfiguration of public space no longer exclusively linked to the state, but as a space of citizen and community participation, proposing the university a mode of working in its relations with the community, which places citizens and stakeholders in the idea of the democratization of knowledge.
\end{abstract}

Key words: university, community relations, public space

\section{Relações universidade com a comunidade: um espaço para a reconfiguração do público}

Resumo: Este trabalho desenvolve uma primeira proposta para resolver a questão da comunidade universitária, como uma área de reconfiguração do público, explicando primeiro uma posição teórica sobre os conceitos espaço público, comunidade e comunidade universitária, para avançar em seguida, as possíveis relações entre eles. Regista as mudanças do século XXI em relação à reconfiguração do espaço público e não exclusivamente vinculados ao estado, mas como um espaço de cidadania e participação da comunidade, propondo uma forma de trabalhar na 
universidade nas suas relações com a comunidade, o que coloca aos cidadãos como protagonistas da idéia de democratização do conhecimento.

blico

Palavras-chave: universidade, relações com a comunidade, o espaço pú-

\section{Introducción}

El espacio de lo público ha estado asociado tradicionalmente al rol del Estado, sin embargo la realidad actual de nuestra región requiere de la participación de los ciudadanos en los temas de la comunidad y en los procesos de decisión y ejecución de esas decisiones, y es de esta manera que el espacio de lo público se conforma como ámbito de participación ciudadana y comunitaria.

En este escenario, cabe a la universidad, considerada como institución social de referencia y pertenencia, un rol protagónico en el desarrollo de relaciones con la comunidad de la cual es parte, con el objetivo de configurar una nueva expresión de lo público en términos de relación entre los sujetos y de espacio de construcción de ciudadanía.

\section{El espacio público}

Para Habermas (1962-1994) los orígenes de la esfera pública se registran en la polis griega considerada como el lugar de los ciudadanos libres; así como en el derecho romano en el cual alcanzó su institucionalización jurídica. Y en este sentido Birules (2005) recordando a Arendt, señala que la polis griega era el único lugar donde los sujetos podían mostrar quienes eran; lo cual deviene de la idea de pluralidad en tanto elemento constitutivo de la condición humana.

La esfera de lo público se caracteriza por diferenciarse de la esfera doméstica y en este sentido se configura como un ámbito de ejercicio de la ciudadanía y de debate de los temas comunes que son preocupación de los ciudadanos; y al respecto Lechner (2003:10) nos alerta acerca de las dificultades que generaría considerar que un listado de problemas privados pudiera conformar un ámbito público.

Para Quiroga (1994:151) lo público es un campo simbólico de deliberación y actuación colectiva de los ciudadanos, preocupados por los asuntos de interés general, que desborda el campo de la esfera política para comprender el dominio de lo social. Este espacio de lo público es tanto un espacio formal (las instituciones), como informal (la sociedad civil), dado que conforma un espacio de comunicación entre los miembros de la comunidad. 
Según Rabotnikof (1993, p.76) lo público puede ser considerado como respuesta a la demanda de la comunidad, como vigencia del estado de derecho o como parte de una cultura política. Y es entonces que la autora señala que: "El espacio de lo público, entendido como el mundo en común, como espacio de aparición y como patrimonio o herencia institucional que constituye esa comunidad debería articular esas carencias generadas por una modernización acelerada, traumática y excluyente”. (Rabottnikof, 1993: 90)

Tradicionalmente asociado al estado y a sus instituciones, en el siglo XXI lo público se ha venido transformando en el campo de ejercicio de la ciudadanía. Y para hablar de ciudadanía es necesario recordar que el concepto ya aparece relacionado al de comunidad en la democracia ateniense durante el siglo V a.C. Sin embargo en la actualidad, la ciudadanía no puede ser simplemente considerada un status legal, definido por un conjunto de derechos y obligaciones; sino que ha de ser considerada también en términos de identidad y de expresión de pertenencia a una comunidad política (Kymlicka, 1996). En este sentido, Castillo García (2007) dice que los procesos de configuración de lo ciudadano, derivan de las maneras en que las personas se relacionan y de las formas en que construyen su vida en comunidad. La idea de comunidad permite visualizar a los individuos como sujetos y en este sentido vale la pena recordar a Lechner (2002) cuando señala que la política es también subjetiva y es un campo de relaciones intersubjetivo de conflicto.

\section{La comunidad}

Cuando hablamos de comunidad, no hablamos de uniformidad, ya que la comunidad implica la inclusión de la diversidad y el logro del compartir en la misma. Porque comunidad, es sinónimo de interacción en lo diferente que es, asimismo, voluntariamente compartido; y entonces hablamos de comunidad en tanto unidad de lo diverso.

Cortina (2006: 3) hace referencia a que los seres humanos nacen y viven en una comunidad concreta en la cual aprenden a valorar y a vivir, y agrega que si el eje social es la comunidad, situada entre el individuo y el Estado; la autonomía personal solo se logra en comunidad, lo cual requiere que el individuo se responsabilice de su comunidad (Cortina, 2001). Pero la noción de pertenencia a una comunidad, no contiene solamente un sentimiento de arraigo, sino que implica un sentimiento de responsabilidad y de lealtad; y es así que la idea de ciudadanía se articula a partir del reconocimiento de que los miembros de la comunidad tienen rasgos que los identifican y que los distinguen de quienes no son parte de ella.

Las definiciones acerca de la comunidad han venido cambiando y reconfigurándose en el tiempo. Algunos autores la han definido como un espacio de construcción de intersubjetividad (Lo Biondo,1999), otros como una red de relaciones de apoyo mutuo (Sarason, 1974), o como instancia 
superadora de lo individual acumulado o repetido (Blanco Abarca, 1988), y otros la entienden como localidad y también como grupo relacional; ya que la primera noción es la que hace referencia al barrio, a la ciudad o a un área rural determinada y la segunda se basa en las relaciones interpersonales más allá de la geografía (Maya Jariego, 2004).

En las últimas décadas en América Latina, las ciudades y sus alrededores han crecido de una manera acelerada y desmesurada, conteniendo en espacios pequeños un gran número de personas que se caracterizan por su heterogeneidad cultural. Al mismo tiempo se ha producido la llegada de poblaciones migrantes de las zonas rurales y/o de países limítrofes, que han tenido tradicionalmente como objetivos buscar trabajo y mejorar las condiciones de vida; de esta manera no sólo han surgido nuevos barrios sino que se han superpoblado los existentes; y a lo largo de este proceso el concepto de comunidad ha venido modificándose.

Este nuevo siglo muestra características tales como la crisis del antiguo modelo de socialización, basado en la sociabilidad barrial y la frecuentación de los espacios públicos, como típicos lugares de encuentro entre los diferentes grupos sociales (Svampa, 2002: 89). Y es entonces que los habitantes de estas ciudades, como dice Seibold (1999: 472473), van aprendiendo a caminar y circular por espacios anónimos (calles, plazas, estaciones de trenes, shoppings, mercados, comercios) lugares diferentes de aquellos que cotidianamente transitaran en sus comunidades de origen, en sus pagos, donde las personas se encontraban e interactuaban.

Frente a estas descripciones la propuesta se centra en recuperar la comunidad como espacio de relaciones cara a cara, regresando al primer significado de la palabra comunitas que mostraba que común era lo no propio, lo que empezaba donde lo propio terminaba, lo que concernía a todos y por lo tanto tenía carácter de público, aquel espacio donde prevalecía la reciprocidad o mutualidad del dar que determinaba un compromiso; y entonces, en el sentido antiguo, el significado de communis era el de quien comparte una responsabilidad y comunitas era el conjunto de personas a quienes las unía un deber en tanto miembros de esa comunidad (Espósito, 2003: 25-31).

\section{La universidad}

La universidad nace en el siglo XII concebida como una asociación dotada de unidad, conformada por grupos de personas cuyos objetivos eran corporativos y sin haber estado asociada necesariamente a un lugar físico. De esta manera, en tanto corporación, la universidad expresaba la organización de sus miembros que defendían el monopolio de enseñar, el cual se encontraba simbolizado en el otorgamiento de la licencia. Las más antiguas universidades de Europa aparecen entre 1180 y 1230 como instituciones supranacionales, y es a partir del siglo XIII que los grupos que comienzan 
a hacerse autónomos dan lugar a la generación de las Facultades, las cuales se conformaron como núcleos, a partir del desarrollo de las diferentes disciplinas.

Con el transcurrir del tiempo, las universidades se fueron independizando paulatinamente del poder político y del eclesiástico, y fue así que a fines de la Edad Media, las grandes universidades se convirtieron en verdaderas potencias políticas, desempeñando un papel activo en las luchas que tuvieron lugar entre los estados, para finalmente integrarse a las nuevas estructuras nacionales de los mismos (Le Goff, 2001, p. 132).

En el siglo XIX surgen en la escena universitaria las primeras acciones que podrían ser considerados los antecedentes de la denominada extensión universitaria, asociadas a la expansión de los ideales de la Revolución Francesa, a la organización de las primeras luchas sociales y al desarrollo de movimientos de vanguardia en lo artístico (Baez Padrón, 2009:349). Y es así que la extensión pasa a ser considerada a fines del siglo XX como una función en el vínculo entre universidad y sociedad en tanto elemento integrador y dinamizador en el que ambas se enriquecen mutuamente (Alarcon, 1994 citado por Baez Padrón, 2009), centrando su accionar en el eje cultural.

Pero en el siglo XXI la universidad ha ampliado su tradicional rol de productora y reproductora de conocimiento científico convirtiéndose en un espacio de interacción social para los sujetos que conviven en ella. Y es entonces que partimos de la idea de concebir a la universidad como una institución social de referencia para los sujetos que viven y transitan en ella su vida cotidiana, lo cual implica considerarla mucho más que un lugar de construcción y reproducción del saber, ya que es verla también como un espacio de socialización y construcción de ciudadanía efectiva. Al decir de Murcia Peña (2009)

La universidad es un escenario social, cultural, político, ético-estético y cognitivo, donde se confrontan constantemente ideas, sentimientos y proyectos, pero sobre todo donde se viven y comparten experiencias, teorías y sensibilidades que pretenden ayudar a mantener, construir y desarrollar al individuo, la sociedad y la cultura (Murcia Peña, 2009: 244).

Los esquemas de inteligibilidad que nos permiten entendernos en la vida universitaria se construyen en interacción entre lo definido por el estado, lo definido por la misma universidad y lo definido por la comunidad (Murcia Peña, 2009, 237). Y es entonces que la universidad como institución, se constituye como escenario para que la ciudadanía pueda construirse y desplegarse.

$\mathrm{Al}$ respecto Cortina (2005: 19) señala que resulta necesario lograr la educación de ciudadanos arraigados, comprometidos con su comunidad local, sabedores de que la ciudadanía los conduce a trabajar por el mundo al que pertenecen como ciudadanos. 
Baez Padrón (2010: 348) identifica tres procesos en el desarrollo de las funciones y actividades de la universidad: el proceso docente-educativo, el proceso de investigación científica y el proceso de transferencia de los conocimientos a la sociedad. Tradicionalmente se ha venido desarrollando un esquema de trabajo universidad-sociedad que se ha basado primordialmente en la transferencia de conocimientos en un esquema de interacción con el medio circundante y si bien esta modalidad ha venido permitiendo un primer contacto y acercamiento al afuera de las instituciones universitarias, el desafío actual se centra en ampliar el trabajo en la construcción de relaciones universidad-comunidad que permitan efectivamente colocar el conocimiento generado por la universidad en respuesta a las necesidades sociales de la población.

Y al hablar de necesidades sociales en el contexto latinoamericano, recordaremos la teoría del desarrollo a escala humana propuesta por Max Neef, Elizalde y Hopenhayn (1986) quienes entienden que las necesidades no son solo la falta de algo (carencia) sino que la potencialidad que eso implica. Asimismo las necesidades tienen un carácter social-universal siendo que lo que cambia de acuerdo a la construcción histórica, son los satisfactores, definidos como "formas de ser, tener, hacer y estar, de carácter individual y colectivo, conducentes a la actualización de necesidades” (Max Neef et al 1986: 41). Los autores apoyan su propuesta en la teoría del protagonismo de las personas en tanto sujetos del desarrollo y no como objetos del mismo, respetando la autonomía y la diversidad, entonces la inclusión del concepto satisfactor como parte del proceso económico, implica considerar lo subjetivo. Y dado que el desarrollo se refiere entonces a las personas y no a los objetos, el mejor proceso de desarrollo será el que permita elevar más la calidad de vida de las personas, dependiendo de las posibilidades que tengan de satisfacer adecuadamente sus necesidades humanas fundamentales.

Esta nueva mirada acerca del rol de la universidad en su relación con la comunidad, que considera la trasmisión de conocimiento científico y a su vez avanza sobre una propuesta de desarrollo humano, coloca al proceso de construcción de ciudadanía en el centro del escenario. Y es entonces que resulta necesario recordar a Sen (2000) cuando expresa que el acceso a la educación y a la salud son las dos oportunidades sociales que cualquier estado que se defina como democrático debe efectivamente garantizar a sus ciudadanos. Desde esta mirada la universidad queda entonces identificada como uno de los espacios públicos de construcción y ejercicio de ciudadanía que se caracteriza por la existencia del debate público y del respeto a las diferencias.

\section{Desarrollando las relaciones universidad-comunidad como un nuevo espacio de lo público}

Partiendo de la consideración de que la universidad es una institución con autonomía, también simultáneamente se reconoce que es una ins- 
titución vinculada a la estructura y características de la sociedad en la cual se desarrolla.

Entonces si la institución universitaria solo fijara su mirada en la vida intramuros, perdería parte de su rol social, aquel que se relaciona con la mirada extramuros. Los tiempos que corren requieren de una universidad comprometida con la vida comunitaria, con las necesidades de los ciudadanos que habitan la comunidad, con el ejercicio de un rol de democratización del saber basado en el principio que reconoce que el conocimiento se construye socialmente y en base al respeto a las diversidades de cada grupo humano.

Si consideramos a la universidad como una organización que es parte de la comunidad e interactúa como una de las actoras sociales de los procesos de desarrollo, en principio sus actividades tendrían que desarrollarse en forma activa no solo dentro de los edificios universitarios sino que en el escenario comunitario. De esta manera, identificaríamos el rol activo de la universidad en la construcción de diagnósticos situacionales de las necesidades de las comunidades, entendiendo por necesidades la idea de Max Neef et al (1986) acerca de que las necesidades son sociales, más que individuales.

Se requiere entonces de decisiones concretas que vinculen efectivamente a la universidad con la comunidad, a partir del desarrollo de acciones conjuntas tales como la construcción de diagnósticos situacionales comunitarios que tengan en cuenta la mirada de los protagonistas y que puedan ser considerados por los organismos estatales de generación de políticas públicas como una información innovadora a las metodologías de tipo tradicional; la sistematización de una guía de recursos de la comunidad que se encuentre en permanente actualización y que sea accesible para su consulta a todos los miembros de la misma; la organización de diferentes cursos e instancias de capacitación destinados a los miembros de la comunidad en relación a temas que consideren de interés, la organización de charlas en las distintas organizaciones de la comunidad sobre temas de preocupación para sus miembros, la generación de un espacio comunitario de formación en investigación y desarrollo para estudiantes de grado y posgrado de la universidad y la edición de publicaciones periódicas, virtuales y gráficas, que muestren las producciones que se generen como resultado de los trabajos desarrollados.

En esta tarea nos encontramos embarcados a partir de la creación de UNI-COM, área de relaciones universidad-comunidad de la Facultad de Ciencias Sociales de la Universidad Nacional de Lomas de Zamora, Argentina. 
Polis, Revista de la Universidad Bolivariana, Volumen 11, $N^{\circ}$ 32, 2012

\section{Nota}

${ }^{1}$ Con mi agradecimiento a las autoridades de la Facultad de Ciencias Sociales de la Universidad Nacional de Lomas de Zamora, Santiago Aragón y Franco Lavolpe, que me han posibilitado organizar y dirigir UNI-COM, relaciones universidad-comunidad y desarrollar así este trabajo. 


\section{Bibliografía}

Baez Padrón, G. (2010), “La participación protagónica estudiantil en el proceso de extensión universitaria”. Revista Latinoamericana de Ciencias Sociales, Niñez y Juventud. Vol. $8 N^{\circ}$ 1. Enero-Junio 2010. CINDE-Universidad de Manizales. Colombia. Pp. 347-362

Birules, F. (2005), “Introducción”. En Arendt, H. ¿Qué es la política? Paidós. ICE/UAB. Bs. As. Pp. 9-40

Blanco Abarca, A. (1988), “La psicología comunitaria, una nueva utopía para final del siglo XX”. En Martin Gonzalez, A, Chacón Fuertes, F y Martinez García, M. Psicología Comunitaria. Editorial Visor. Madrid. Pp. 11-33.

Castillo García, J. (2007), “La configuración de las ciudadanías en estudiantes universitarios y universitarias de pregrado en Manizales, Colombia”. Revista Latinoamericana de Ciencias Sociales, Niñez y Juventud, Manizales. Vol 5. $N^{\circ}$ 2. Julio-Diciembre 2007. CINDE-Universidad de Manizales. Colombia. Pp. 755-810

Cortina, A. (2001), Alianza y contrato. Editorial Trotta. Madrid.

Ídem (2005), La misión de la universidad: educar para la ciudadanía del siglo XXI. Conferencia de inauguración del año académico. Pontificia Universidad de Valparaíso. Chile. 30 de marzo.

Ídem (2006), Ética, ciudadanía y modernidad. Conferencia en la Universidad de Valparaíso. Chile. Mimeo

Esposito, R. (2003), Comunitas. Origen y destino de la comunidad. Amorrortu editores. Bs. As.

Habermas, J. (1962, 1994), The Structural Transformation of the Public Sphere. Cambridge, Polity Press.

Kymlicka, W. (1996), Ciudadanía multicultural. Paidós. Barcelona

Lechner, N. (2002), Las sombras del mañana. La dimensión subjetiva de la política. IOM Ediciones. Santiago de Chile.

Le Goff, J. (2001), Los intelectuales en la Edad Media. Gedisa. Barcelona.

Lo Biondo, G. (1999), “Algunos elementos del aporte de Bernard Lonergan a la política”. En Scanonne, J y Santuc V. (comp.) Lo político en América Latina. Editorial Bonum. Bs. As. pp.281-302

Max Neef, M. , Elizalde, A. y Hopenhayn, M. (1986), Desarrollo a escala humana. CEPAUR, Suecia 
Maya Jariego, I. (2004), "Sentido de comunidad y potenciación comunitaria”. Apuntes de Psicología, Vol. 22, número 2, págs. 187-211.Colegio oficial de Psicología de Andalucía Occidental- Universidad de Sevilla.

Murcia Peña, N. (2009), "Vida universitaria e imaginarios: posibilidad en definición de políticas de educación superior”. Revista Latinoamericana de Ciencias Sociales, Niñez y Juventud. Vol. 6, $N^{\circ}$ 2. Enero-Junio 2009. CINDE- Universidad de Manizales. Colombia. Pp. 235-266.

Quiroga, H. (1996), Esfera pública, política y ciudadanía. Revista Internacional de Filosofía Política 7. UNED-Universidad Autónoma Metropolitana. Barcelona. Pp 141-158.

Rabotnikof, N. (1993), Lo público y sus problemas: notas para una reconsideración. RIFP 2. UNED. Barcelona. Pp 75-98

Sarason, S. B. (1974), The psychological sense of community: prospects for a community psychology. San Franscisco: Jossey Bass.

Seibold, J. (1999), “Ciudadanìa, transformación educativa e imaginario social urbano”. En Scanone, J,. y Santuc, V. (comp. ) Lo político en América Latina. Editorial Bonum. Bs. As. Pp. 463-511.

Svampa, M. (2002), "Las nuevas urbanizaciones privadas, socialidad y socialización: la integración social hacia arriba”. En Beccaria, L, Feldman, S, Gonzalez Bombal, I. Kessler, G. Murmis, M. y Svampa, M. Sociedad y socialidad en la Argentina de los 90. Universidad Nacional de General Sarmiento. Editorial Biblos. Bs. As. Pp. 55-95.

Tonon, G. (2009) (comp.), Comunidad, participación y socialización política. Espacio Editorial. Bs. As.

Ídem (2010), “Pensar indicadores para la construcción de las políticas: el rol de la universidad”. Libro del Congreso Compromiso social y calidad educativa: desafíos de la extensión. UNCU (editora). Mendoza. 